\title{
Psychoanalytisches Arbeiten in Institutionen - am Beispiel des «Pinocchio», einer Beratungsstelle für Eltern und Kinder in Zürich
}

\author{
Michael Frei (Zürich) und Norbert Wolff(Wetzikon)
}

Zusammenfassung: Darstellung der Geschichte und Arbeitsweise in der psychoanalytisch orientierten Beratungsstelle für Eltern und Kinder "Pinocchio» in Zürich. Ausgehend vom Anspruch sowohl ein niederschwelliges Angebot für die Eltern mit zeitnahen Beratungsterminen zu machen, als auch das psychoanalytisch orientierte Verstehen der Beziehungsdynamik innerhalb der Familie und die Wiederaufnahme der progressiven Entwicklung des Kindes in den Fokus der gemeinsamen Arbeit zu stellen, wird zunächst die Gründungsidee der Beratungsstelle und anschliessend die Arbeitsweise der psychoanalytisch ausgebildeten Mitarbeitenden dargestellt. Anhand einer kurzen Fallvignette werden das psychoanalytische Fallverständnis und die entwicklungsorientierte Interventionstechnik exemplarisch dargestellt.

Schlüsselwörter: Psychoanalytische Pädagogik, psychoanalytische Haltung in der Beratung, Elternarbeit, psychoanalytisch-entwicklungsorientierte Begleitung von Kindern

\section{$1 \quad$ Einleitung - Entwicklung ermöglichen}

«Pinocchio» ist eine niederschwellige psychoanalytische Beratungsstelle in Zürich für Eltern mit Kindern im Alter von zwei bis vierzehn Jahren. Diese wenden sich mit Sorgen oder Unsicherheiten bezüglich der Entwicklung und Erziehung ihres Kindes (oder ihrer Kinder) an uns. Im Zentrum der Beratung steht das Wohl und die psychische Entwicklung des Kindes. Um diese unterstützen zu können, arbeiten wir eng mit den Eltern zusammen. Kindergarten, Schule, Hort oder andere für das Kind wichtige Personen beziehen wir nach Rücksprache mit den Eltern in unsere Arbeit ein. 
Die Beratungsstelle entstand 1982 aus einem Projekt der Schule für Soziale Arbeit Zürich. Federführend in diesem Projekt war Heidi Urben, die während ihrer Tätigkeit in städtischen Horteinrichtungen immer wieder Kinder mit psychosozialen Auffälligkeiten beobachtete. Dies motivierte sie einerseits, Sozialpädagogik zu studieren, andererseits schrieb sie sich am Psychoanalytischen Seminar Zürich (PSZ) ein und absolvierte ihre eigene Analyse. Diese Ausbildung legte den Grundstein der theoretischen und praktischen Ausrichtung der Beratungsstelle, die heute mit sieben Angestellten besetzt ist.

Finanzielle Unterstützung erhielt die Gründerin vom Marie Meierhofer Institut für das Kind (MMI). Marie Meierhofer wurde als städtische Kinderärztin in den 1940er Jahren - ähnlich wie Heidi Urben 40 Jahr später - auf Bindungsschwierigkeiten und erschwerte Entwicklungsbedingungen von Kindern in Tages- und Wocheneinrichtungen der Stadt aufmerksam. 1957 gründete sie das «Institut für Psychohygiene im Kindesalter», welches 1978 nach ihr benannt wurde. Das MMI ist bis heute eine für die ganze Schweiz bedeutsame Forschungs- und Lehrinstitution im Früh- oder Kleinkindbereich und dem «Pinocchio» nach wie vor fachlich und freundschaftlich verbunden.

Ziel des Projekts von Heidi Urben war es, eine Anlaufstelle zu schaffen, in der Eltern und Kinder als eigenständige Subjekte mit ihren individuellen Wünschen, Ängsten und Konflikten gewürdigt und respektvoll begleitet werden. Idée fondatrice der Institution war, dass unabhängig von den finanziellen Voraussetzungen jedes Kind ein Recht darauf hat, in seiner Entwicklung Hilfe und Unterstützung zu erfahren. Dies sollte im Namen der Institution abgebildet werden, womit die bekannte Kinderbuchfigur von Carlo Collodi (1826-1890) ins Spiel kommt: Der arme Holzschnitzermeister Gepetto bekommt einen verzauberten Holzklotz geschenkt, der zu ihm spricht. Daraus schnitzt er sich einen Hampelmann, den er auf den Namen Pinocchio, auf Italienisch ungefähr: kleiner Holz-Dummkopf, tauft. Pinocchio lernt laufen, er lacht und weint und büxt seinem Schöpfer aus. Die Vater-Sohn-Beziehung wird durch seine Entwicklung arg geprüft. Er macht Dummheiten, lügt und bereitet seinem Vater grosse Sorgen. Es entfaltet sich die mäandrierende und ereignisreiche Geschichte der Menschwerdung Pinocchios, der, hin- und hergerissen zwischen Trieben und Idealansprüchen, seinen Weg in die Welt und unter die Menschen finden muss. Vater und Sohn drohen dabei auf je eigene Weise verloren zu gehen und müssen sich im Verlauf der Geschichte wiederfinden und versöhnen. Die psychoanalytisch-entwicklungspsychologischen Bezüge des Märchens sind offensichtlich. 


\section{$2 \quad$ Entwicklung und Organisation der Institution}

Rechtlicher Träger der Beratungsstelle ist der 1983 gegründete und steuerbefreite Verein «Pinocchio», dessen Vorstand immer mit einer praktizierenden Psychoanalytikerin ${ }^{1}$ besetzt war. Diese verantwortet die Einhaltung der psychoanalytischen Ausrichtung der Beratungsstelle, die in den Vereinsstatuten gesetzt wird. ${ }^{2}$ Der Vorstand arbeitet ehrenamtlich und kümmert sich um die strategische Ausrichtung sowie die rechtlichen Belange.

Als erstes Anstellungskriterium neuer Beraterinnen des «Pinocchio» gilt bis heute eine abgeschlossene und eidgenössisch anerkannte psychoanalytisch-psychotherapeutische Ausbildung. Dazu gehört ein abgeschlossenes Hochschulstudium in klinischer Psychologie und Psychopathologie, eine eigene Psychoanalyse Freud/scher Richtung sowie begleitend eine Ausbildung oder Berufserfahrung im Kleinkind- und/oder im Schulbereich. In dieser Hinsicht verfügt das Beratungsteam des «Pinocchio», im Vergleich mit anderen, ähnlich ausgerichteten Beratungsdiensten, über einen hohen Ausbildungsstand. Seine explizit psychoanalytische Ausrichtung ist praktisch ein Alleinstellungsmerkmal. Für die Beraterinnen wird die Teilnahme an den vierzehntäglichen Teamsupervisionen durch erfahrene Kinderanalytikerinnen sowie regelmässige Intervisionen und die persönliche Einzelsupervision, ebenfalls bei niedergelassenen Psychoanalytikerinnen, vorausgesetzt.

Was den «Pinocchio» bis heute ausmacht, ist ein langjährig konstantes Team, das nach wie vor im Sinne der Gründungsidee arbeitet und sich im Zusammenhang mit den sozio-ökonomischen und wissenschaftlichen Entwicklungen kritisch mit den Rahmenbedingungen seiner Arbeit auseinandersetzt: Wie können wir, angesichts der Schwierigkeiten, denen Familien und Kinder heute ausgesetzt sind, möglichst niederschwellig und gleichwohl fachlich fundiert (psychoanalytisch) arbeiten?

Während die Beratungsstelle bis in die 2000er Jahre stark interdisziplinär geprägt war - es waren Sozialpädagoginnen, Ethnologinnen und Psychologinnen, alle mit psychoanalytischer Ausrichtung, vertreten - liessen sich nach und nach fast nur noch Psychologinnen bzw. Psychotherapeutinnen finden, welche den verlangten psychoanalytischen Ausbildungsstand mitbrachten. Gesellschaftspolitische und rechtliche Umwälzungen führten dazu, dass sich in anderen Disziplinen der Sozialwissenschaften kaum mehr potentielle Mitarbeiterinnen mit einem psychoanalytischen Hintergrund finden lassen. ${ }^{3}$

«Pinocchio» ist heute, 37 Jahre nach seiner Gründung, zu einem relevanten Ansprechpartner für Familien, Krippen, Kindergärten, Schulen und Hort- 
einrichtungen, aber auch für Opferberatungsstellen, Sozialdienste und Kinderschutzinstitutionen sowie der Kinderärztinnen und der Kinder- und Jugendpsychiatrischen Poliklinik der Stadt Zürich (KJPP) geworden. Offizielle Stellen aus Stadt und Kanton wenden sich bei entsprechenden Fragen und mit allfälligen Überweisungen an uns. Es ist überaus erfreulich, dass eine so kleine, psychoanalytisch orientierte Institution so lange bestehen und sich als unabhängige Anlaufstelle für Eltern und Kinder bewähren konnte. Elisabeth von Salis hat die Geschichte der Beratungsstelle und Aspekte ihrer Arbeitsweise bereits im Journal Nr. 50 vorgestellt (von Salis, 2009). An der konzeptionellen Ausrichtung hat sich nichts geändert. Das Angebot hat sich jedoch in Form verschiedener Projekte und Kooperationen erweitert. Wir betrachten diese Projekte als soziale Interventionen, mit denen wir - im Rahmen unserer Möglichkeiten - auf sozio-ökonomische Entwicklungen zu antworten suchen. Dabei tragen diese Projekte essenziell zur Vernetzung im Feld der uns umgebenden Institutionen bei.

\section{Das Angebot - niederschwellige psychoanalytische Beratung}

«Pinocchio» bietet ratsuchenden Eltern bei sämtlichen Fragen und Unsicherheiten zur Erziehung und zur psychischen Entwicklung ihrer Kinder seine Unterstützung an. Wir beraten Eltern und begleiten Kinder vor dem Hintergrund unserer psychoanalytischen Erfahrung entwicklungsorientiert. Eltern erreichen uns mit einer grossen Bandbreite von Anliegen. Die Anfragen reichen von einfachen, erzieherischen Unsicherheiten im Familienalltag zu Problemen der Emotionsregulation, Schulschwierigkeiten und Überforderung der Eltern, bis hin zu schweren psychischen Entwicklungs- und Bindungsstörungen, anhaltend prekären Familienumständen und Zuweisungen über involvierte Ämter.

Das Angebot richtet sich auch an Fachpersonen (wie Lehrerinnen usw.). Vordringliches organisatorisches Ziel ist es, möglichst rasch und unkompliziert auf eine Anfrage reagieren zu können. In der Regel finden wir innerhalb von ein bis zwei Wochen einen ersten Beratungstermin. Das Erstgespräch wird nach Möglichkeit pauschal mit CHF 50 verrechnet. Die Kosten für alle weiteren Beratungsgespräche werden auf Basis des versteuerten Einkommens der Eltern vereinbart. Gemäss der Gründungsidee besteht jedoch der institutionelle Anspruch, dass kein Kind aus finanziellen Gründen auf unsere Unterstützung verzichten muss. Der «Pinocchio» bietet stark reduzierte Sozialtarife an und kann seine Arbeit auch aufnehmen, wenn die Finanzierung nicht im Voraus gesichert ist.

Das Angebot ist dahingehend eingeschränkt, dass «Pinocchio» keine psychologischen Gutachten zur parteilichen Verwendung vor Gericht erstellt. 
Bei zerstrittenen Elternpaaren ist es unser vordringliches Anliegen, mit allen Beteiligten zugunsten des Kindes ins Gespräch zu kommen. Bei Fällen, die uns über offizielle Stellen wie z. B. die Kindes- und Erwachsenenschutzbehörden (KESB) zur Beurteilung vermittelt werden, versuchen wir eine möglichst unabhängige Stellungnahme im Sinne des Kindswohls zu leisten. Des Weiteren nehmen wir keine testpsychologische Begutachtung vor und unsere Arbeit kann nicht über die Krankenkassen abgegolten werden.

Krankheitswertige Fälle triagieren wir an unser Netzwerk von psychoanalytischen Psychotherapeutinnen und Psychiaterinnen. Neben der Elternberatung bietet die Beratungsstelle auch entwicklungsorientierte Begleitungen von Kindern bis 14 Jahre im Einzelsetting an. In diesem Artikel soll der Schwerpunkt jedoch auf der Elternarbeit liegen.

\section{Elternarbeit im Zentrum - ohne Eltern geht es nicht}

Am Anfang der Kontaktaufnahme steht in der Regel die Sorge der Eltern. Nicht selten stehen diese jedoch unter Druck von aussen, sei es von der Schule oder einer Behörde. Die Kontaktaufnahme erfolgt daher nicht immer ganz freiwillig, was eine sorgfältige Beachtung des Beziehungsaufbaus gebietet. Aber auch bei hoher Eigenmotivation der Eltern ist es wichtig, dem Aufbau einer vertrauensvollen Beziehung besondere Aufmerksamkeit zu schenken. In dieser Hinsicht ist z. B. die typische Situation, wenn Eltern zu Beginn einer Beratung nach Tipps für schwierige Erziehungssituationen fragen, tückisch. Ratschläge, insbesondere vor dem Aufbau eines tragfähigen Arbeitsbündnisses, können problematische Konsequenzen für die Entwicklung der Beziehungsdynamik zwischen der Beraterin und den Eltern haben. Eltern können zudem, aufgrund der ambivalenten Einstellung zum Problem und zur Beratungssituation, einen Rat häufig nicht umsetzen, da dessen Erfolg die eigene Unfähigkeit bestätigen würde. Auch bei anders gelagerter Übertragungskonstellation ist dessen Erfolgsaussicht, ohne geteiltes Verständnis der Funktion des Symptoms und ohne eine entsprechende Veränderung der familiären Beziehungen, gering (Figdor, 1999, S. 36).

Unsere Haltung den Eltern gegenüber soll durch das Bemühen um ein gemeinsames Verständnis der gegenwärtigen Herausforderungen geprägt sein. Für seine Entwicklung ist das Kind auf starke und zufriedene Eltern angewiesen. So richten sich unsere Anstrengungen in der Elternarbeit darauf, diese dabei zu unterstützen, ihren erzieherischen Aufgaben «good-enough» nachzukommen und ihre Beziehung zu sich und zum Kind liebevoll zu entspannen (Winnicott, 1997, S. 13). In diesem Sinn können durchaus psychoedukative Elemente in die Beratung ein- 
fliessen. Grundlage der Zusammenarbeit ist das «diagnostische Arbeitsbündnis», in dem die Eltern als «Experten ihres Kindes» angesprochen und dazu eingeladen werden, gemeinsam die «Gründe für die Probleme zu verstehen», um sie dabei unterstützen zu können, «das Richtige zu tun» (Figdor, 1999, S. 54). Pedro Grosz formuliert als «Zweck der Elterngespräche in der psychoanalytischen Arbeit» die Aufgabe, «einen Veränderungsprozess in Gang zu bringen, der allen [...] in der Familie eine Annäherung und Verständigung ermöglicht. Damit entsteht eine neue emotionale Entwicklung» (2018, S. 30).

«Pinocchio» bietet auf unkompliziertem Weg einen vertraulichen Rahmen zur Erarbeitung eines gemeinsamen Verständnisses der Problematik, derentwegen sich die Eltern sorgen oder an uns verwiesen wurden. Wir legen den Fokus auf die altersgemässen Entwicklungsaufgaben des Kindes, bzw. auf die Umstände, die eine progressive Entwicklung behindern. Wir werden dabei nicht primär als Therapiestelle für Kinder wahrgenommen und verstehen uns auch nicht als solche. Im Vordergrund unserer Arbeit steht die Unterstützung und die (Wieder-)Befähigung der Eltern. Nicht in jedem Fall ist es notwendig und hilfreich, das Kind zu sehen, und der Wunsch der Eltern nach Vorstellung des Kindes ist in jedem Fall sorgfältig zu prüfen (Grieser, 2018; Grosz, 2018; von Salis 2009). Die prinzipielle Möglichkeit, bei entsprechender Indikation jedoch auch langfristig im Einzelsetting mit Kindern arbeiten zu können, bürgt für die Beratungsstelle und fördert so das Vertrauen in unser Angebot.

In Abgrenzung zur Arbeit in einer Praxis ist das Setting, im Hinblick auf die Beratung der Eltern und die allfällige Begleitung des Kindes, initial offener. Die Eltern wissen oft selbst nicht genau, was sie sich wünschen und ob sie sich für ihr Kind eine Behandlung vorstellen können. Dieser Umstand kann sich auch als Chance erweisen. In Abgrenzung zu medizinischen und (schul-)psychologischen Diensten wird das Kind nicht von Beginn an als Indexpatientin gesetzt. Es sollen hier immer erst die Eltern, ihre Beziehung zum Kind und zum sozialen Umfeld der Familie betrachtet werden. Im «Pinocchio» wird auch keine Diagnose gestellt. Nicht die Symptome zählen, sondern vielmehr die Bedeutung des Erlebens und Verhaltens des Kindes, die familiären Beziehungen sowie - im Sinne der psychoanalytischen Pädagogik - die gesellschaftlichen Anforderungen an die Familie als Kleingruppe (Datler, Figdor \& Gstach, 1999).

Wir interessieren uns für die Herausforderungen, welche die Entwicklungsbedürfnisse des Kindes, sein Temperament sowie seine Erlebens- und Verhaltensweisen an die Familie stellen. Vor dem Hintergrund psychoanalytischer Entwicklungstheorien, der Bindungsforschung, einem relationalen Psychothe- 
rapieansatz und dem psychoanalytisch-systemischen Verständnis zwischenmenschlicher Beziehungen, versuchen wir, gemeinsam mit den ratsuchenden Eltern, Entwicklungsblockaden zu erkennen und zu überwinden. Erkenntnisse der Psychoanalyse sollen so für pädagogisches Handeln nutzbar gemacht werden. Daher greift das Konzept einer Erziehungsberatung mit der Fachperson als Expertin und den Eltern als Ratsuchenden zu kurz. Wir wollen mit den Eltern in ein «diagnostisches Arbeitsbündnis» einsteigen und ein gemeinsames Verständnis der aktuellen Situation erarbeiten (Figdor, 1999, S. 48f.).

Im Allgemeinen findet das Erstgespräch mit beiden Eltern und ohne Kind statt. In der Mehrzahl der Fälle treten erst die Mütter mit uns in Kontakt. Es ist uns jedoch wichtig, die Väter von Anfang an in die Beratung mit einzubeziehen. Wenn immer möglich wird das Erstgespräch mit beiden Elternteilen gemeinsam durchgeführt. So schlägt auch Grieser als «Standardsetting» vor, «immer beide Eltern gemeinsam zu sehen, wenn dem nicht unüberwindbare Schwierigkeiten im Wege stehen» (2018, S. 27).

Im Erstgespräch geht es darum, die Problemstellung und die verschiedenen Perspektiven der Beteiligten möglichst gut zu erfassen. Die umfassende Klärung des problematisierten Verhaltens des Kindes, die Überlegungen der Eltern zu dessen Genese und ihre Erwartungen und Ziele helfen uns, die familiäre Dynamik zu verstehen und gemeinsam zu überlegen, wie wir die Eltern und ihr Kind am besten unterstützen können. Die Klärung bewusster und unbewusster Motive, Ambivalenzen und Widerstände, sowie die Analyse der Gegenübertragung sind für dieses Verständnis unabdingbar. Die Beraterin fragt sich unter anderem, wie es ihr als Kind in dieser Familie gehen würde. Je nach Situation kann diese «Abklärungsphase» auch mehrere Gespräche umfassen. Die sorgfältige Abklärung zu Beginn der Behandlung ist uns wichtig, auch im Hinblick auf eine allfällige Triage (Bürgin \& Steck, 2013; Novick \& Novick, 2009).

In einem zweiten Schritt, und nur bei entsprechender Indikation, laden wir das Kind ein. Über altersentsprechendes Spiel und Gespräch lernen wir sein Erleben der Situation kennen. Danach machen wir einen Vorschlag zum weiteren Vorgehen: sei dies eine entwicklungsorientierte Begleitung des Kindes mit regelmässig stattfindenden Elterngesprächen, sei dies eine ausschliessliche Erziehungsberatung der Eltern oder ein anderes der Familiensituation angepasstes Vorgehen. Die Form legen wir mit den Eltern fest und gleichen sie im Verlauf mit dem Entwicklungsprozess des Kindes und der Familie ab. Die Dauer unseres Engagements kann zwischen einem bzw. einigen wenigen Beratungsgesprächen nur mit den Eltern, bis zu einer langfristigen Begleitung mit wöchentlichen Sit- 
zungen mit dem Kind und regelmässigen Elterngesprächen über mehrere Jahre variieren. Auf Wunsch kann der «Pinocchio» den Kontakt zwischen konflikthaft getrennten Eltern, oder bei Konflikten zwischen den Eltern und involvierten Institutionen, im Sinne des Kindswohls triangulieren und vermitteln.

Unsere beraterische Haltung lässt sich dahingehend zusammenfassen, dass wir die Sorgen der Eltern ernst nehmen wollen. Wir gehen grundsätzlich davon aus, dass sie es gut mit dem Kind meinen und versuchen ihr Bestes zu geben, gegenwärtig aber nicht über adäquatere Mittel verfügen. Es geht uns darum, die bestehenden Schwierigkeiten zu verstehen und niemanden zu verurteilen. Dies soll den Boden für ein tragfähiges Arbeitsbündnis bereiten. Erziehungsberatung so verstanden stellt für alle Beteiligten ein Lernfeld dar, keine Korrektur- oder Disziplinierungsmassnahme. Es steht nicht die unmittelbare Veränderung des Verhaltens, die «Symptomfreiheit», im Vordergrund, sondern die Veränderung von Beziehungsqualitäten sowie ein Zuwachs an sozialen und emotionalen Kompetenzen.

Dem Beratungsteam ist die Berücksichtigung kulturspezifischer Besonderheiten wichtig (Grieser, 2018, S.53), und wir gehen davon aus, dass pädagogisches und therapeutisches Handeln immer auch wertegeleitet ist. Figdor schreibt, dass man seinen «Rat auch vor sich selbst, vor seinem eigenen Wert- und Normsystem verantworten können» muss (1999, S. 35). Uns geht es in der Regel um die verbesserte soziale Teilhabe und Integration des Kindes bzw. der Familie sowie um harmonischere innerfamiliäre Beziehungen. Damit anstehende Entwicklungsaufgaben gemeistert werden können, ist oft eine Erweiterung des Handlungsspielraums nötig. Theoretisch stützen wir uns auf die Konzepte der psychoanalytischen Pädagogik, wie sie beispielsweise Datler, Figdor und Gstach unter dem Titel «Die Wiederentdeckung der Freude am Kind» (1999) umrissen haben. Im manifesten Verhalten des Kindes zeigen sich latente Wünsche, Ängste und Konflikte der ganzen Familie. Daher ist es unabdingbar, etwas von der unbewussten Dynamik einer Familie zu verstehen. Die Eltern werden als Expertinnen ihres Kindes angesprochen und zu einem Prozess der gemeinsamen Erarbeitung der Gründe seines Erlebens und Verhaltens eingeladen.

Das Ziel unseres Engagements besteht in der «Wiederaufnahme der progressiven Entwicklungslinien» (Novick \& Novick, 2009, S. 33) sowie der Wiederherstellung der Eltern-Kind-Beziehung als Quelle von Zärtlichkeit, Sicherheit und Vertrauen. «[D]ie von Schuldgefühlen durchdrungene Angst» soll «in konstruktive Sorge und Anteilnahme» (ebd., S. 79) umgewandelt werden. Der über das vereinbarte Setting gesetzte Rahmen ermöglicht, mitunter heftige Gefühle der Wut, 
Trauer und Enttäuschung über Nicht-Erreichtes, Verlorenes und Erlittenes auszuhalten und als Container für diese zu dienen (Bion, 1962). Wir möchten den Eltern zur «haltenden Umwelt» werden und suchen ihnen die «Ich-Stützung» zu bieten, die sie zur Erfüllung der elterlichen Rolle benötigen (Winnicott, 2002, S. 71). Für den Anspruch, eine niederschwellige Elternberatung anzubieten, sind «Erinnern, Wiederholen und Durcharbeiten» (Freud, 1914g) natürlich Fernziele. Damit die bekannten, maladaptiven Interaktionsmuster jedoch nicht zwanghaft wiederholt werden müssen, dienen uns diese Konzepte gleichwohl als Orientierung.

\section{$5 \quad$ Fallbeispiel - David und die Angst vor Beziehungsverlust}

Im Fokus unserer Arbeit steht die Veränderung familiärer Beziehungen im Dienste einer guten Entwicklung des Kindes. Dazu ein Beispiel:

Frau M. wandte sich in der Sorge, den liebevollen Kontakt zu ihrem älteren Kind zu verlieren an uns. Im Erstgespräch äusserte sie Angst davor, dass ihr der 10-jährige Sohn «abhanden kommen» könnte und sie sich «entfremden» würden. Heftiger Streit häufte sich zwischen ihnen, weil er ihre Aufforderungen, etwas zu erledigen nicht gleich befolgte, worauf sie ungehalten reagierte. Frau M. lebte seit über einem Jahr getrennt vom Vater ihrer zwei Kinder. Zur Trennung kam es, nachdem sie festgestellt hatte, dass ihr Mann eine Aussenbeziehung führte, welche er nicht aufgeben wollte. Frau M. war von diesem Vertrauensbruch zutiefst erschüttert und fühlte sich während Monaten emotional labil. Sie erlebte sich als dünnhäutig und reagierte bei Konflikten mit ihren Kindern impulsiv. Insbesondere mit dem älteren Sohn, der sie in vielem an den Vater erinnerte, geriet sie häufig aneinander. Sie hatte "keine Nerven» und schämte sich wegen ihres Schreiens und Drohens.

Nachdem ihr Bemühen, eine gute Mutter zu sein, die sich im Alltag sehr für die Belange der Kinder engagierte, diese in vielen Bereichen unterstützte (u.a. in den Peerkontakten und Hobbys) und ihnen, trotz der eigenen Traurigkeit aufgrund der Trennung, ein schönes Zuhause bieten wollte, vom Berater gesehen und gewürdigt wurde, wurde es in der Beratung möglich, ihre Verletzung durch das Verhalten des Mannes anzusprechen. Dieser bemühte sich, den Kindern an den Wochenenden ein guter Vater zu sein, der aller- 
lei mit ihnen unternahm und dafür geschätzt wurde. Die Mutter fühlte sich im Alltag jedoch sehr alleine, bei schulischen Belangen wenig unterstützt und zuweilen in der Erziehung überfordert. Ihre Enttäuschung über den Mann nagte an ihrem Selbstwert. Frustration über den anstrengenden Alltag und die knappen finanziellen Verhältnisse äusserten sich in einer bisher nicht gekannten Reizbarkeit. In der Beratung konnte gemeinsam erarbeitet werden, dass nicht nur sie, sondern auch die Kinder, mit der Trennung und den damit einhergehenden Veränderungen zu kämpfen hatten. Das gemeinsame Verstehen der anspruchsvollen, neuen familiären Situation ermöglichte es der Mutter, von übertriebenen Erwartungen an die Selbständigkeit und Kooperation der Kinder Abstand zu nehmen. Dank der veränderten Haltung und dem vertieften Verständnis für die Bedürfnisse ihrer Kinder entspannte sich das Zusammenleben schliesslich erheblich. Gemeinsames Spielen und Spass haben fand wieder Platz und die perfektionistischen Ansprüche an ein reibungsloses Funktionieren, um die eigenen Ressourcen zu schonen, traten zurück. Möglich war dies in Folge eines gemeinsamen Verständnisses dessen, was die Mutter in ihrer eigenen Entwicklung geprägt hatte und durch die Anerkennung der grossen Anforderungen, die sie aktuell zu bewältigen hat.

Die Reflexion des Übertragungsgeschehens im Beratungssetting, insbesondere die Analyse der Gegenübertragung, half dem Berater, wesentliche Stolpersteine auf dem Weg zu einem besseren Verständnis der Situation zu erkennen und sich nicht vorschnell zu Ratschlägen verführen zu lassen: $\mathrm{Zu}$ Beginn der Beratung zeigte die Mutter die Tendenz, über das Verhalten des Sohnes und das Betragen des Vaters zu klagen, wodurch sich der Berater gut in den Sohn einfühlen konnte, der sich gegen den Druck des Reibungslos-funktionieren-müssens und die «Opferhaltung» der Mutter wehrte. Die Herausforderung bestand dann einerseits darin, sowohl der reflexartigen «Solidarisierung» mit dem Sohn als auch der Abwehrhaltung gegenüber der «nervigen Mutter» gewahr zu werden (den unbewussten Tendenzen dank der eigenen Analyse auf die Schliche zu kommen), andererseits aber auch die Kränkung und Verletzung der Mutter anzuerkennen, welche diese beständig zu eskamotieren suchte.

Eine pädagogische Antwort auf das unkooperative Verhalten des Sohnes hätte zunächst dem manifesten Beratungsauftrag der Mutter ganz entsprochen, 
das analytische Verständnis der Familiendynamik wäre dabei allerdings aussen vor geblieben. Im Beratungssetting wurde das familiäre Übertragungsgeschehen reinszeniert. Dank der Erarbeitung eines gemeinsamen Verständnisses dessen, was hier (Beratung) wie dort (Familie) zur Aufführung kam, konnte eine Entspannung und Vertiefung der Mutter-Sohn-Beziehung sowie die persönliche Entwicklung beider auf positive Weise unterstützt werden.

Im vorgestellten Fall war es ausreichend, mit der Mutter zu arbeiten, ohne das Kind oder den Vater miteinzubeziehen. Die Mutter wollte ihre Erwartungen an den Sohn mit einer Fachperson reflektieren. Sie sah ihren Beitrag an der Konflikteskalation, da es zwischen ihr und dem Sohn immer dann besser lief, wenn sie entspannt und ausgeruht war. Die Mutter litt unter ihrem Erleben der Situation und ihren Kontrollverlusten. Sie wünschte vor allem für sich Unterstützung. In der Beratung sah sie bald einen Zusammenhang zwischen ihrem Leid und der Kränkung durch den Ex und Vater der Kinder. Sie erkannte die Gefahr einer Übertragung negativer Gefühle von ihm auf den Sohn. Der Einfluss weit zurückliegenderVerletzungen in ihrer Herkunftsfamilie, in welcher sie sich weder gesehen noch geschätzt fühlte, konnte aufgedeckt, in der Beratung wiedererlebt und ansatzweise bearbeitet werden. Es gelang Frau M. zunehmend, mehr Verantwortung für ihr Handeln zu übernehmen. Im beraterischen Verständigungs- und Verstehensprozess war hier insbesondere das Konzept der «verantworteten Schuld» (Figdor, 1999, S. 49) hilfreich: Infolge der Anerkennung eigener Verletzungen, welche sie durch ihren Vater erlitten hatte, konnte auch der Anteil «ihrer Schuld» an der elterlichen Trennung angesprochen werden. Frau M. konnte die Klärung zulassen, dass sie als Eltern mit der Trennung den Kindern etwas zugemutet haben, was sich diese nicht gewünscht haben. Die elterliche Unterstützung der Kinder im Trauerprozess ist besser möglich, wenn eigene Anteile an der veränderten Familiensituation anerkannt und ausgehalten werden. Frau M. konnte gegenüber den Kindern ihren Teil der Verantwortung dieses Scheiterns übernehmen und musste nicht mehr alle Schuld dem Mann anlasten. Infolge der positiven Entwicklung des Mutter-Sohn-Verhältnisses in dieser mehrmonatigen Elternberatung drängte sich keine Erweiterung des Settings auf. Der Einbezug des Sohnes im Sinne einer entwicklungsorientierten Begleitung und/oder des Vaters in Form von gemeinsamen Eltern- oder Einzelgesprächen wäre bei einem anderen Verlauf durchaus möglich gewesen.

\section{6}

\section{Schlussfolgerungen}

Die psychoanalytische Beratungsstelle «Pinocchio» stellt eine Bereicherung des Beratungs- und Therapieangebotes der Stadt Zürich dar. Ihr Angebot ist zwar 
im Sinne eines unbürokratischen Zugangs und kurzer Wartezeit niederschwellig. Da die Eltern jedoch einen einkommensabhängigen Beitrag leisten, der bei durchschnittlichem Einkommen doch deutlich höher als der Selbstbehalt bei der Krankenkasse ausfällt, ist das finanzielle Engagement der Eltern im Vergleich zu einer therapeutischen Intervention hoch. Dass dennoch langfristige Beratungen und Begleitungen möglich sind, spricht für die Attraktivität des Angebots.

Es wurde dargestellt, dass das Prinzip der Niederschwelligkeit nicht im Widerspruch zum Anliegen, psychoanalytisch arbeiten zu wollen, stehen muss. Unsere Beratungsstelle ist für viele Eltern eine erste Anlaufstelle bei Sorgen, welche zum gegebenen Zeitpunkt oder aus anderen Gründen (noch) nicht den Weg in eine psychotherapeutische Praxis oder psychiatrische Institution gefunden haben. Das offene Angebot und das Bemühen um ein gemeinsames Verständnis der Situation sprechen die Eltern an. Im Verlauf ist es wichtig, über sorgfältige Elternarbeit ein Setting zu etablieren, das ausreichend Sicherheit und Kontinuität für die Beratung der Eltern und/oder die Begleitung des Kindes bietet. Eltern wie Kinder finden im «Pinocchio» einen vertraulichen Rahmen, in dem etwas verstanden und verändert werden kann. Blockierte Entwicklungen kommen wieder in Gang. Insbesondere Kinder, die in prekären Verhältnissen aufwachsen, finden hier eine langfristig zuverlässige und vertrauenswürdige Unterstützung, was auch von den zuweisenden Stellen geschätzt wird. Kinder aus allen Verhältnissen finden Gehör, bevor ihre Schwierigkeiten krankheitswertige Störungen werden müssen. In diesem Sinne ist das Angebot auch eine Ergänzung des Präventivbereichs der psychosozialen Versorgung von Familien in und um Zürich.

\section{Literatur}

Bion, W. R. (1962). Learning from Experience. London: Heinemann.

Bürgin, D. \& Steck, B. (2013). Indikation psychoanalytischer Psychotherapie mit Kindern und Jugendlichen:Diagnostisch-therapeutisches Vorgehen und Fallbeispiele. Stuttgart: Klett-Cotta.

Datler, W., Figdor, H. \& Gstach, J. (Hrsg.) (1999). Die Wiederentdeckung der Freude am Kind. Psychoanalytisch-pädagogische Erziehungsberatung heute. Giessen: Psychosozial Verlag.

Datler, W. (1999). Erziehungsberatung und die Annahme eines dynamischen Unbewussten. Über zentrale Charakteristika psychoanalytisch-pädagogischer Erziehungsberatung. In W. Datler, H. Figdor \& J. Gstach (Hrsg.), Die Wiederentdeckung der Freude am Kind. Psychoanalytisch-pädagogische Erziehungsberatung heute (S. 11-31). Giessen: Psychosozial Verlag. 
Figdor, H. (1999). Aufklärung, verantwortete Schuld und die Wiederentdeckung der Freude am Kind. Grundprinzipien des Wiener Konzeptes psychoanalytisch-pädagogischer Erziehungsberatung. InW. Datler, H. Figdor \& J. Gstach (Hrsg.), Die Wiederentdeckung der Freude am Kind. Psychoanalytisch-pädagogische Erziehungsberatung heute (S. 32-60). Giessen: Psychosozial Verlag.

Freud, S. (1914g). Erinnern, Wiederholen und Durcharbeiten. Weitere Ratschläge zur Technik der Psychoanalyse. GWX, S. 126-136.

Grieser, J. (2018). Elternarbeit in der Psychotherapie von Kindern und Jugendlichen. Göttingen: Vandenhoeck und Ruprecht.

Grosz, P. (2018). Einblicke - Kinderpsychoanalytische Falldarstellungen und die dazu gehörenden Elterngespräche. Zürich: Vissivo Verlag.

Novick, J. \& Novick, K. M. (2009). Elternarbeit in der Kinderpsychoanalyse. Klinik und Theorie. Frankfurt a. M.: Brandes \& Apsel.

von Salis, E. (2009). Die psychoanalytisch orientierte sozialpädagogische Beratungsstelle Pinocchio. Journal für Psychoanalyse. 50. 131-137.

Winnicott, D. W. (2002). Reifungsprozesse und fördernde Umwelt. Giessen: Psychosozial-Verlag.

Winnicott, D. W. (1997). Playing and Reality. London: Tavistock Publications

\section{Anmerkungen}

1 Der besseren Lesbarkeit halber wird in diesem Artikel ausschliesslich die weibliche Form verwendet.

2 Die Statuten des Vereins «Pinocchio» nennen unter $§ 4.1$ die «psychoanalytisch basierte Beratung und Begleitung von Kindern und Eltern» als Aufgabe der Beratungsstelle.

3 In dieser Zeit wurde das Psychologieberufegesetz umgearbeitet, wodurch die psychoanalytische Ausbildung für Studienabgehende anderer Fakultäten deutlich an Reiz verlor.

\section{Angaben zu den Autoren}

Michael Frei, Dr. phil., Fachpsychologe für Psychotherapie FSP, 1982. Master of Advanced Studies in Psychoanalytic Psychotherapy USZ (MASP). Assistenz und Doktorat am Lehrstuhl für Klinische Psychologie, Psychotherapie und Psychoanalyse von Frau Prof. B. Boothe, Universität Zürich, 2010-2013. Geschäftsleiter der Beratungsstelle für Eltern und Kinder «Pinocchio» in Zürich seit 2017. Delegierte und selbständige Praxis in Zürich seit 2013.

Norbert Wolff, Dipl. Psych., Fachpsychologe für Psychotherapie FSP, 1963. Psychotherapeutische Weiterbildung am PSZ, bei Prof. D. Bürgin, bei Prof. M. Buchholz, am Schweizer Institut für Psychotraumatologie (SIPT), bei 
Dr. A. Krüger (PITT-KID) . Mitarbeiter in der Beratungsstelle für Eltern und Kinder «Pinocchio» in Zürich seit 2002. Delegierte und selbständige Praxistätigkeit in Bubikon seit 2013. 